\title{
GENE SRY ET ANOMALIES DE LA DETERMINATION GENETIQUE DU SEXE CHEZ L'HOMME
}

\author{
Françis POULAT, Catherine GOZE, Brigitte BOIZET, Philippe BERTA
}

\author{
Centre de Recherche de Biochimie Macromoléculaire \\ INSERM U 249 - CNRS UPR 9008 - Route de Mende, B.P. 5051, 34033 Montpellier Cédex
}

\begin{abstract}
SRY GENE AND HEREDITARY ERRORS OF GENETIC SEX DETERMINATION IN MAN. Normal sexual development in man is the consequence of a complex process. This review focuses on the translation of genetic sex (XX or XY karyotype) into gonadal sex (testis or ovary). During the last three years attempts to identify and clone the testis determining factor (TDF) have exploited detailed maps of the $\mathrm{Y}$ chromosome established by geneticists over the last decade. A candidate gene, named SRY (sex determining region, Y) located at the tip of the short arm of the $Y$ chromosome, shows many characteristics in common with TDF in that it is the sole element of the $\mathrm{Y}$ chromosome required for male development. The discovery of TDF led us to analyse sex-reversed individuals, i.e. $X X$ males and $X Y$ females, with the aim of constructing a model for the processes regulating the development of an organ as complex as the testis. This SRY gene is now the subject of intense molecular biological effort by various groups, effort which we hope will elucidate the mechanism(s) of sex determination. Key words : Sex determination, Testis determining factor, SRY, Sex reversal. Andrologie, 1992, 2 : 50-52.
\end{abstract}

Dans l'étude des mécanismes liés à la reproduction chez les mammifères et donc chez l'homme, la compréhension du mode de détermination du sexe (mâle versus femelle), base de cette même reproduction sexuée, a toujours occupé une place particulière.

Comme formulé très tôt par Alfred Jost (6), le développement sexuel normal d'un embryon de mammifere résulte d'un processus séquentiel à 3 étapes.

La première étape implique l'établissement du sexe génétique. La capacité de réalisation des caryotypes a rapidement démontré l'existence d'un dimorphisme chromosomique: ainsi si le sexe hétérogamétique $(X Y)$ est mâle, le sexe homogamétique (XX) est femelle.
Dans une deuxième étape, le sexe génétique est alors traduit en un sexe gonadique. Par une série d'évènements encore incompris aujourd'hui, l'information génétique va permettre la transformation d'une gonade jusqu'alors indifférenciée (ou gonada primordia identique chez tout embryon jusqu'à environ la 6ème semaine de développement chez l'homme) en testicule chez le mâle ou en ovaire chez la femelle.

L'étape terminale traduira ce sexe gonadique en un sexe phénotypique, ceci en fonction des hormones émises par les deux types de gonades. Dans cette étape, se mettront en place aussi bien les tractus génitaux internes ou externes que les caractéristiques fonctionnelles ou comportementales liées à chaque sexe. Ainsi, l'appareil génital interne se développera soit à partir des canaux de Wolf chez l'homme, ou à partir des conduits de Muller chez la femme, ces deux types co-existant chez l'embryon avant le choix du type de gonade.

En absence d'un testicule, comme chez la femelle ou dans un embryon mâle après castration, le développement du sexe phénotypique sera femelle. La masculinisation quant à elle résulte d'une action positive des hormones testiculaires alors que la féminisation est un processus passif qui ne semble pas requérir d'action hormonale dans la gonade foetale.

La détermination du sexe proprement dite correspond à la première étape, c'est à dire celle traduisant un sexe chromosomique en un sexe gonadique.

L'analyse caryotypique d'individus porteurs d'un syndrome de Turner de sexe femelle et en majorité de composition gonosomique $\mathrm{XO}$ ainsi que celle d'individus porteurs d'un syndrome de Klinefelter pouvant être XXY ou encore XXXY par exemple et de sexe mâle a convaincu les généticiens de l'existence d'un facteur dominant localisé sur le chromosome $Y$ induisant la masculinisation indépendemment du nombre de chromosomes X.

Ce facteur fut très tôt dénommé TDF, acronyme anglais pour Testis Determining Factor. Sa localisation sur le chromosome $Y$ fut alors entre- prise. Les grandes étapes de cette recherche sont résumées sur la figure 1. Celle-ci fut ralentie par la particularité même du chromosome $\mathrm{Y}$, à savoir son unicité qui lui fait échapper aux règles de la génétique mendélienne classique excluant ainsi toute génétique réverse ou pour être plus à la mode, positionnelle.

Figure 1 : Evolution de la localisation de la fonction TDF sur le chromosome $\mathrm{Y}$ dans les dernières décennies.

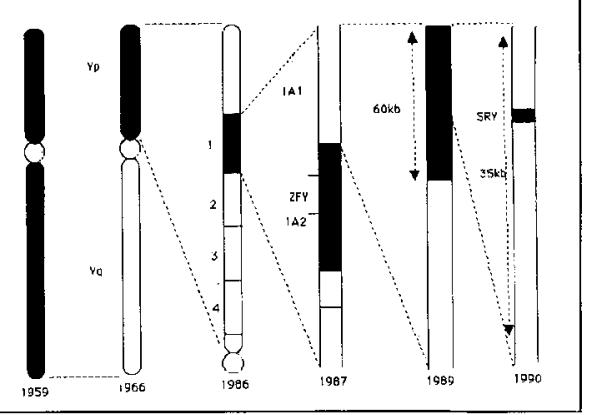

Trois types de cartes du chromosome $\mathrm{Y}$ furent alors réalisées :

a - Une carte méiotique: en effet, il existe quant même à l'extrémité du bras court du chromosome $Y$ une zone d'identité commune à l'extrémité du bras court du chromosome $X$. Ceci permet à chaque événement méiotique l'association des chromosomes $\mathrm{X}$ et $\mathrm{Y}$, association permettant leur parfaite ségrégation à l'anaphase. Il est intéressant de noter que cette association s'accompagne d'un événement de recombinaison unique mais obligatoire. Cette région d'identité qui, malgré sa localisation sur les chromosomes sexuels, n'apparaît pas liée au sexe, a été dénommée pseudoautosomale. Ce n'est donc pas dans cette région que TDF se trouvera localisé.

b - Une carte de délétion: l'établissement de cette carte spécifique au chromosome $\mathrm{Y}$ a été permise par l'existence de rares anomalies lors de l'association entre le chromosome $\mathrm{X}$ et $\mathrm{Y}$ au cours de la méiose. On constate en effet à des fréquences de l'ordre de 1/20000 que l'échange décrit cidessus entre ces deux chromosomes ne se limite plus à leur zone d'identité mais affecte des portions chromosomiques liées au sexe. On arrive ainsi à la production de deux types d'individus :

- les hommes XX: ceux-ci dans leur grande majorité ont hérité un chromosome $\mathrm{X}$ d'origine paternelle "enrichi" en un fragment de chromoso- 
me Y sur son bras court. Si ce dernier inclut la fonction TDF, il en résulte alors un tel phénomène de réversion sexuelle,

- les femmes XY: a fortiori un chromosome $Y$ en quelque sorte "débarrassê" de ce TDF engendre une forme de réversion sexuelle particulière, les femmes $X Y$ porteuses d'une dysgénésie gonadique pure.

$c$ - Une carte de restriction sur des grands fragments d'ADN qui a permis de relier les deux cartes précédentes.

De nombreux gènes candidats furent alors publiés, mais nous ne les détaillerons pas ici. On peut cependant mentionner l'avant-dernier candidat, le gène ZFY (10) (figure 1). Si ce gène localisé sur le chromosome $Y$ code pour un facteur de transcription à doigts de zinc, divers arguments ont pu l'écarter dans cette fonction de détermination du sexe. On s'oriente plutôt pour un rôle au cours de la spermatogénèse de la protéine ZFY. Il a fallu en fait attendre 1989 pour qu'une étapeclé soit franchie (11). Ainsi une nouvelle catégorie, d'hommes à caryotype $46, \mathrm{XX}$ a pu être décrite. Certains d'entre eux ne sont en effet porteurs que d'une fraction minimale de chromosome $\mathrm{Y}$ sur leur chromosome $\mathrm{X}$ d'origine paternelle. Cette fraction limitée à 35 kilobases, qui exclut le gène ZFY, suffit donc pour engendrer le développement testiculaire que nous qualifierons de somatique. TDF doit obligatoirement s'y trouver localisé.

Une recherche systématique dans cette séquence et reposant sur deux critères que sont la spécificité $Y$ du fragment testé et sá conservation sur le chromosome Y d'un panel de mammifêres permit le clonage d'une séquence de 669 nucléotides (15).

Ce gène monoexonique (par définition, un exon correspond à la partie d'un gène réellement transcrite) fut dénommé SRY (pour "Sex Determining Region, Y"). Trois types d'évidence furent produites pour s'assurer de l'identité entre SRY et le facteur de détermination du sexe porté par le chromosome Y.

- un profil d'expression partiel du gène sry murin a été réalisé; il apparaît que ce gène est transcrit au bon moment (11,5 jours d.p.c. chez l'embryon murin) et au bon endroit (les cordons génitaux) (7).

- l'analyse génétique de femmes XY à dysgénésie gonadique pure. Environ $20 \%$ d'entre elles résultent de mutations ponctuelles spontanées au coeur même de la séquence SRY $(1,5)$. Un seul changement de base sur les 3.109 bases de notre génome suffit donc à induire un changement de phénotype aussi radical que celui d'un homme pour une femme.

- enfin, plus récemment (8), l'introduction d'un fragment d'ADN génomique de 14 kilobases comprenant le gène sry murin a permis par transgénose la production de souris mâles malgré un caryotype XX.

Le mode d'action de la protéine SRY reste encore aujourd'hui énigmatique. Les données physiologiques nous apprennent que la chaîne réactionnelle initiée par le produit du gène SRY conduit à la mise en place d'une catégorie de cellules particulières ou cellules de Sertoli. Connaissant le point de départ, il apparaît intéressant de décrypter les étapes lui succédant.

Le gène SRY code pour une protéine de 223 acides aminés présentant un motif de 80 acides aminés d'homologie partielle à la famille des protéines de haute mobilité (15). Ceci permit d'attribuer une fonction putative de liaison à l'ADN pour la protéine SRY. Nous avons ainsi pu avec d'autres $(4,9,13)$, démontrer la capacité de SRY à lier une séquence nucléotidique courte riche en A-T. La nature exacte de cette séquence reste à préciser. De plus, l'utilisation de la technique de microinjection de fibroblastes embryonnaires humains nous a permis, outre de démontrer la localisation nucléaire de SRY, de préciser la nature du signal permettant sa translocation vers le noyau. La protéine SRY constitue donc bien une protéine nucléaire aux propriétés voisines de celles d'un facteur de transcription. Il reste à souligner une propriété particulière dans I'interaction SRY-ADN: des techniques fluorescentes démontrent la liaison de la protéine au niveau du petit sillon de l'ADN $(3,13)$, caractéristique originale pour un facteur de transcription.

Cependant, le faible degré de spécificité dans la liaison de SRY à l'ADN rend difficile la recherche de ses gènes cibles.

Sur le plan clinique, la classification des désordres du développement sexuel encore connus sous le nom d'ambiguités sexuelles reste très sommaire. Il faut cependant distinguer des erreurs dans la mise en place du sexe gonadique qui seules nous intéressent ici, et celles dans l'établissement du sexe phénotypique d'origines hormonales.

La découverte du gène SRY n'a pas révolutionné bien sûr l'approche clinique des patients de la première catégorie. Outre son utilisation à des fins de sexage (fécondation in vitro à risques), elle permet cependant d'entrevoir un début de classification sur des bases génétiques des anomalies de la mise en place de la gonade.
Les résultats les plus probants dans ce sens sont donnés par les femmes à caryotype $46, \mathrm{XY}$ ou encore par les hommes de caryotype 46,XX.

\section{Femmes XY}

Victimes d'aménorrhée primaire, les femmes $X Y$ présentent une absence de gonade, ou dysgénésie gonadique, et sont observées à une fréquence d'environ 1/100000.

Les études moléculaires en cours révèlent leur hétérogénéité. Seules $20 \%$ d'entre elles présentent une mutation au coeur du gène SRY et plus exactement au niveau de son motif de liaison à l'ADN.

On parlera alors de dysgénésie gonadique pure, ceci étant confirmé par l'absence de tout résidu séminifere en histologie au niveau de leurs gonades fibreuses (streak). La majorité des réversions de ce type reste à expliquer. On peut postuler, dans ces cas, de l'altération d'un ou de plusieurs gènes localisés dans la chaîne réactionnelle en aval du gène SRY. Certaines de ces femmes, outre leur réversion sexuelle, ont en association divers types de syndromes pédiatriques, tels des syndromes rénaux.

Le développement coordonné des tractus génital et urinaire amène à penser à l'utilisation de gènes communs pour leurs mises en place au cours de la vie embryonnaire. Ainsi, le syndrome de Denys-Drash associe-t-il chez des filles XY une tumeur de Wilms. Il a ainsi pu être démontré récemment la présence dans ces cas précis de mutations ponctuelles sporadiques dans les exons 8 et 9 de WTl (12). Cela constitue l'un de nos arguments pour impliquer ce gène dans la cascade de différenciation gonadique.

Une néphropathie particulière peut être également associée à la réversion sexuelle. Nous avons ainsi pu distinguer récemment ce syndrome du précédent sur le plan moléculaire $(2,14)$. Des patientes porteuses de ce syndrome, dit de Frasier, ont été collectées et d'éventuelles délétions chromosomiques sont à l'étude.

\section{Hommes XX}

Les hommes 46,XX sont également hétérogènes quant à leur phénotype. Si la grande majorité d'entre eux résultent bien de la présence d'un fragment de chromosome Y sur leur X paternel, ils peuvent être divisés en sous-classes en fonction de la taille du fragment $Y$ transféré. Nous achevons la caractérisation moléculaire précise (définition des points de cassure) de la classe ne présentant qu'une fraction de 35 kilobases, et ce travail nous permet de revenir sur le dogme selon lequel plus on observerait un nombre d'anomalies phénotypiques faibles, plus le fragment $Y$ présent serait important. 
En conclusion, la découverte du gène SRY procure au biologiste un point de départ dans l'étude de la cascade réactionnelle conduisant, à partir d'une gonade indifférenciée, à un testicule. On conçoit l'importance d'une telle recherche dans le domaine de la biologie du développement.

Les premières données récemment publiées sur SRY n'ont pas encore permis d'en comprendre ni la fonction exacte, ni la nature des cibles génétiques. Cette fois encore, c'est l'étude génétique de différentes formes d'ambiguité sexuelle qui devrait permettre la localisation de nouveaux gènes de détermination du sexe. Enfin, il n'est pas surprenant de constater la grande conservation des gènes de type SRY au cours de l'évolution, mais également l'existence de gènes de type SRY (ou gènes SOX) dont SRY serait le parangon, et qui pourraient jouer un rôle similaire dans la mise en place d'autres grandes fonctions de l'organisme.

\section{RÉFÉRENCES}

1 - Berta P, Hawkins JR, Sinclair AH et al. : Genetic evidence equating SRY and the testis determining factor. Nature, 1990, 348:448-450.

2 - Berta P, Morin D, Poulat F et al. : Molecular analysis of the sex determining region from the $Y$ chromosome in two patients with Frasier syndrome. Horm. Res., 1992, (sous presse).

3 - Giese K, Cox J, Grosscheld R :The HMG domain of lymphoid enhancer factor 1 bends DNA and facilitates assembly of functional nucleoprotein structures. Cell, 1992, 69:185-195.
4 - Harley VR, Jackson DI, Hextall PJ et al. : DNA binding activity of recombinant SRY from normal males and XY females. Science, 1992, 255:453-456.

5 - Hawkins JR, Taylor A, Berta P et al. : Mutational analysis of SRY: nonsense and missense mutations in $X Y$ sex reversal. Human Genetics, 1992, 88:471-474.

6 - Jost A. : A new look at the mechanisms controlling sex differenciation in mammals. Johns Hopkins Med. J., 1972, 130:38.

7 - Koopman P, Munsterberg A, Capel B, Vivian N, Lovell-Badge R. : Expression of a candidate sex determining gene during mouse testis differenciation. Nature, 1990, 348:450-452.

8 - Koopman P, Gubbay J, Vivian N, Goodfellow P, Lovell-Badge R. : Male development of chromosomally female mice transgenic for sry. Nature, 1991, 351:117-121.

9 - Nasrin N, Buggs C, Kong XF, Carnazza I, Goebl $\mathrm{M}$, Alexander-Bridges M. : DNA binding properties of the product of the testis-determining gene and a related protein. Nature, 1991, 354:317-320.

10 - Page DC, Mosher R, Simpson EM et al. :The sex determining region of the human $\mathrm{Y}$ chromosome encodes a fingerprotein. Cell, 1987, 51:1091 1104.

11 - Palmer MS, Sinclair AH, Berta P et al. : Genetic evidence that $\mathrm{ZFY}$ is not the testis determining factor. Nature, 1989, 342:937-939.

12 - Pelletier J, Bruening W, Kashtan CE et al.: Germline mutations in the Wilm's tumor suppressor gene are associated with abnormal urogenital development in Denys-Drash syndrome. Cell, 1991, 67:437-447.
13 - Poulat F, Guichard G, Girard F, et al. : Nuclear localization and DNA binding activities of the testis determining SRY gene product. Cell, 1992, (soumis pour publication).

14 - Poulat F, Morin D, Konig A, et al. : Distinct molecular origins for Denys-Drash and Frasier syndromes. Hum. Genet., 1992, (soumis pour publication).

15 - Sinclair AH, Berta P, Palmer MS et al. : A gene from the human sex determining region encodes a protein with homology to a conserved DNA binding motif. Nature, 1990, 346:245-250.

RESUME : La compréhension des mécanismes de détermination du sexe chez l'homme a franchi une étape importante ces trois dernières années. Le gène SRY, localisé sur le chromosome $Y$, et seul élément nécessaire de ce chromosome au développement mâle, a ainsi pu être isolé. De nombreux critères permettent aujourd'hui de l'assimiler au facteur TDF (ou "testis determining factor"). Par l'étude des propriétés de la protéine SRY et par l'étude d'autres formes d'ambiguités sexuelles, on espère aujourd'hui pouvoir progresser dans la reconstitution de la cascade conduisant à la formation d'un organe aussi complexe que le testicule. On conçoit le formidable challenge que cette recherche constitue dans le domaine de la biologie du développement. Mots-clés : Détermination du sexe, TDF, SRY, Réversions sexuelles. Andrologie, 1992, 2 : 50-52.

\section{CERTIFICAT EUROPEEN D'ANDROLOGIE}

Les universités de Bâle (Dr B. Leibundgut), Fribourg (Pr U. Wetterauer et Pr H. Wokalek) et Strasbourg (Dr A. Clavert) se sont associées afin de réaliser, dans le cadre de l'université du Rhin Supérieur, un enseignement d'Andrologie.

Chaque université prend en charge une partie de l'enseignement. Les cours sont donnés en anglais. Le contrôle de connaissance consiste en une dissertation écrite dans la langue du pays.

Pour tous renseignements, contactez : Docteur A. Clavert

Hospices Civils de Strasbourg

1, place de l'Hôpital - 67091 Strasbourg Cedex

Tél. : 88.36.52.70 